\title{
Understanding the nuances of microwave ablation for more accurate post-treatment assessment
}

\author{
Christy Kim*,1 \\ ${ }^{1}$ AngioDynamics, Inc., 1850 Mt Diablo Blvd, Ste 660, Walnut Creek, CA 94596, USA \\ *Author for correspondence: ckim@angiodynamics.com
}

\begin{abstract}
Microwave ablation (MWA) is a relatively new thermal modality for minimally invasive procedures compared with radiofrequency ablation. Although MWA and radiofrequency ablation are thermal modalities, their underlying physics and principles greatly differ. Consequently, it is imperative that clinicians be aware of how these differences impact realized ablation volumes to consistently ensure technical success and better patient outcomes. This paper will review the nuances specific to MWA technology (i.e., tissue properties, perfusion/heat sink effect, ablation assessment, imaging accuracy and tissue contraction) that are often overlooked based on familiarity with conventional thermal modalities to guide more accurate assessment of post-treatment MWA volumes.
\end{abstract}

First draft submitted: 28 December 2017; Accepted for publication: 29 January 2018; Published online: 14 February 2018

Keywords: ablation assessment $\bullet$ heat sink effect $\bullet$ imaging accuracy $\bullet$ microwave ablation $\bullet$ microwave ablation devices $\bullet$ radiofrequency ablation $\bullet$ thermal ablation $\bullet$ tissue contraction

Microwave ablation (MWA) has recently become a very popular thermal modality for minimally invasive procedures. Although radiofrequency ablation (RFA) has the longest history of accumulated data as the prototypical thermal ablation technique, MWA is quickly gaining momentum because of its technological advancements, including faster ablation times, larger ablation volumes, effectiveness in many tissue types and an improved convection profile [1-4]. Because the mechanism of action of MWA is distinct from RFA, the assessment of MWA volumes may differ from the conventional methods used to assess RFA volumes. Understanding the nuances of MWA technology over other thermal modalities is critical to determine strategies to more accurately assess post-treatment MWA volumes.

\section{MWA is a different thermal ablation than RFA}

Even though MWA and RFA both destroy tissues via thermally mediated coagulative necrosis, MWA and RFA are broadly distinct because of their inherently different mechanisms of energy deposition (Figure 1). RFA is limited in tissues of low electrical conductivity because RFA relies on resistive heating through an electrically conductive path (Figure 1B) [5]. The radiofrequency (RF) electrode is not the source of the heat itself but instead, generates frequencies of $450-500 \mathrm{kHz}$ to frictionally heat up the tissue immediately adjacent to the electrode [5]. The RFA volume is further transmitted by thermal conductance (Figure 1D) [6]. RFA's mechanism of heating necessitates a slow and methodical energy deposition for controlled heating $50-100^{\circ} \mathrm{C}$ to avoid charring and vaporization, which could compromise electrical current flow because desiccated tissue acts as an insulating 'sleeve' around the electrode to limit energy transfer resulting in smaller ablations [5].

In contrast to RFA's primary reliance on thermal conduction, MWA applies electromagnetic fields with higher frequencies of $915 \mathrm{MHz}$ to $2.45 \mathrm{GHz}$ to directly heat tissue to lethal temperatures greater than $150^{\circ} \mathrm{C}$ through dielectric hysteresis [4]. Dielectric hysteresis is a process in which polar molecules, primarily water are forced to continuously realign with the oscillating electric field (Figure 1A). This results in the generation of kinetic energy and subsequent heat generation [4]. Since MWA relies predominately on direct heating over thermal conductance (Figure 1C), MWA has the potential to produce faster and larger ablation volumes with less susceptibility to the heat sink effect, the perfusion mediated cooling provided by local blood vessels, compared with RFA [3,6-8].

Future 8 Medicine 


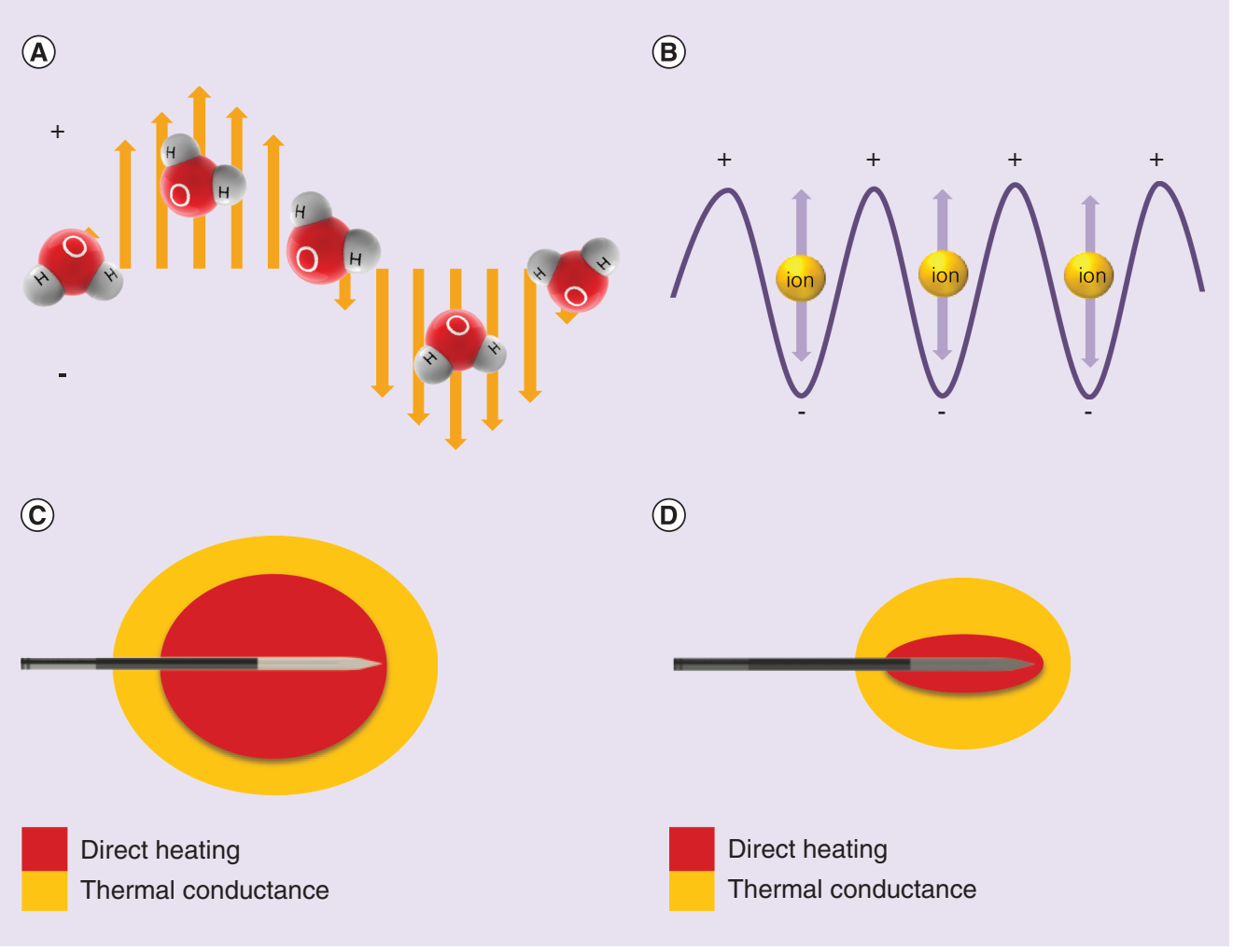

Figure 1. Underlying mechanism of action and heat transfer for microwave ablation and radiofrequency ablation. Microwave ablation (A \& C); radiofrequency ablation (B \& D).

\begin{tabular}{|c|c|c|c|}
\hline Parameter & MWA & RFA & Ref. \\
\hline Tissue properties & + & ++ & {$[4,9,10]$} \\
\hline Perfusion/heat sink & + & ++ & {$[1,6-8,11-13]$} \\
\hline Ablation assessment & + & + & {$[8,14-16]$} \\
\hline Imaging accuracy & ++ & + & [17-19] \\
\hline Tissue contraction & ++ & + & [20-22] \\
\hline
\end{tabular}

+ Sensitive; ++ More sensitive.

MWA: Microwave ablation; RFA: Radiofrequency ablation.

Moreover, MWA in comparison to RFA is capable of propagating through many tissue types despite having high impedance such as in the lung or within charred tissue [4,9]. Given that MWA and RFA achieve different temperature thresholds through varying energy deposition and kinetics, post-treatment imaging using MWA may need to be assessed differently than previously assumed with RFA technology. Table 1 summarizes the parameters that have been shown to affect ablation volumes and assessment using MWA and RFA technologies that will be further explored in this review.

\section{Each MWA system is uniquely different}

Strengthened by continual advancements in microwave technology, MWA device manufacturers are advancing minimally invasive techniques at a rapid pace with the help of innovative clinicians. However, there are nuances of MWA technology that every clinician must be aware of to effectively plan, perform and evaluate procedural success to ensure that lesions are completely treated with sufficient ablative safety margins $(0.5-1 \mathrm{~cm})$.

Every MWA system consists of a generator, a cable and an applicator; however no two MWA systems are alike because these components will vary by manufacturer according to their respective intellectual property. Power is generated from the microwave generator which can have varying frequencies of $915 \mathrm{MHz}$ (low frequency) or 
$2.45 \mathrm{GHz}$ (high frequency). MWA systems operating at $2.45 \mathrm{GHz}$ generally result in greater power deposition and larger ablation volumes than those operating at $915 \mathrm{MHz}$, resulting in larger ablations in a shorter period of time [2,11,23-25]. The cable distributes the power from the generator to the applicator tip and the degree of power conservation will also vary by design; therefore, the operating generator wattage should not be confused with the applied power at the applicator tip [24]. Most importantly, every MWA system is unique because of the careful consideration taken into the microwave applicator design with respect to geometry, diameter and cooling mechanism. Consequently, every MWA system will have a characteristic ablation zone shape and size that will be further scalable by power and time settings [24]. Typical MWA systems require $10 \mathrm{~min}$ and some systems even require multiple applicators to reach the desired maximum ablation volumes [24,26,27]. To minimize cost, time required for positioning additional applicators, and the higher risk of bleeding from additional penetrations, a one applicator MWA system that can create up to a $5 \mathrm{~cm}$ ablation in minimal time would be ideal to ensure adequate safety margins (i.e., Acculis MTA System; Microsulis Medical, Denmead, England) [24]. In summary, generalizations about MWA technology must be refined per MWA device and not misleadingly across all similarly classified technologies because of the range in design specifications across manufacturers.

\section{Ex vivo MWA validations versus in vivo MWA results}

Recently, there have been studies evaluating manufacturers' reference values provided with the device's instructions for use and how ex vivo ablation volumes are significantly larger than ablation volumes obtained from in vivo animal and diseased human tissues [12,27-29]. Ex vivo animal models are limited by insufficiently mimicking in vivo tissue conditions of perfusion, temperature, cellular morphology, anatomy and tissue contraction. In vivo imaging of ablation volumes or technical success is limited by visual interpretation of the imaging modality of choice and the kinetics of dynamic tissue ablation volumes with respect to time in a physiological environment. Nonetheless, ablation volumes derived from ex vivo animal models are valuable in that they provide a rough approximation of ablation volumes to be expected in practice.

Winokur et al. conducted a clinical study performing percutaneous MWAs using the AMICA (HS Medical, FL, USA) and Certus PR applicators (NeuWave Medical, WI, USA) and found that they produced ablation volumes with significant differences in the short and long axis diameters and resultant volumes compared with the manufacturer's ex vivo reference values (note: the short axis diameter tends to be the limiting factor in an ablation because it has to encompass the tumor along with a safety margin) [28]. This study raises concerns about translating manufacturers' ex vivo derived ablation volumes into clinical practice. However, some of the shortcoming of this study include heterogeneity of tissue types, relatively small sample size ( $\mathrm{n}=25$ and 12$)$, the misuse of the manufacturers' recommended directions for use through the use of serial ablations (eight of 25 and nine of 12) and oversimplification of the kinetics of varying power and time by combining both into a single independent variable. Power and time should not be combined because an equivalent total applied energy of $30 \mathrm{~kJ}$ using in vivo porcine livers produces significantly different volumes if applied at $25 \mathrm{~W}\left(23.7 \pm 26.5 \mathrm{~cm}^{3}\right)$ or $100 \mathrm{~W}$ $\left(265.7 \pm 208.1 \mathrm{~cm}^{3}\right)$ [30]. In addition, simultaneous power delivery has been shown to have differing ablation shapes and sizes in comparison to sequentially applied microwave energy [26].

When ablation measurements are analyzed by manufacturer's power and time settings, more insights pertaining to specific MWA systems may be made on how accurate ex vivo data approximate in vivo data. Table 2 includes the in vivo and ex vivo short and long axis diameter comparisons extrapolated from the study conducted by Winokur et al. for the AMICA and Certus PR applicators at varying power and time settings. Surprisingly, for the AMICA applicator, the MWA long axis diameter $(\mathrm{p}=0.799)$ and short axis diameters $(\mathrm{p}=0.105)$ were not statistically different across all time points tested at 40,60 and $80 \mathrm{~W}$ between in vivo and manufacturer's ex vivo data (note: time points were selected based on availability of manufacturer's reference data). However, the average relative difference of the short axis diameter of the in vivo data to the ex vivo data with the AMICA applicator showed a $22.9 \pm 17.4 \%$ decrease whereas the long axis diameter decrease was only $2.7 \pm 16.9 \%$ (Table 2 ). Figure $2 \mathrm{~A} \& \mathrm{~B}$ show the in vivo versus ex vivo comparison of both the short and long axis ablation diameters obtained with the AMICA applicator at $60 \mathrm{~W}$. Intriguingly, MWAs with the Certus PR at $65 \mathrm{~W}$ showed a similar conservation of long axis diameter $(\mathrm{p}=0.223)$ and short axis diameter $(\mathrm{p}=0.142)$ across ex vivo and in vivo studies but with average smaller long axis diameters $(17.7 \pm 4.8 \%)$ in addition to average smaller short axis diameters $(23.2 \pm 1.2 \%)$ in conservation with the AMICA applicator (Table 2, Figure 2C \& D). Although this analysis is limited in sample size and statistical significance, these results suggest that ablation dimensions vary in vivo versus ex vivo and to what extent will depend on the MWA system's specific applicator design, power and time settings, and tissue property. 
Table 2. Summary of average percent differences of in vivo short and long axis diameters relative to ex vivo references for the AMICA and Certus PR Microwave Ablation Systems at various power and time settings.

\begin{tabular}{|c|c|c|c|c|c|c|c|c|c|}
\hline \multicolumn{2}{|c|}{ Settings } & \multicolumn{4}{|c|}{ Short axis diameter } & \multicolumn{4}{|c|}{ Long axis diameter } \\
\hline Power (W) & Time (min) & In vivo (mm) & Ex vivo $(\mathrm{mm})$ & $\begin{array}{l}\% \text { Diff. (rel. to } \\
\text { ex vivo) }\end{array}$ & Avg $\%$ diff. & In vivo $(\mathrm{mm})$ & Ex vivo $(\mathrm{mm})$ & $\begin{array}{l}\% \text { Diff. (rel. to } \\
\text { ex vivo) }\end{array}$ & Avg \% diff. \\
\hline \multicolumn{10}{|l|}{ AMICA } \\
\hline 40 & 5 & 16.3 & 29 & -43.8 & $-22.9 \pm 17.4$ & 36.3 & 36 & 0.8 & $-2.7 \pm 16.9$ \\
\hline 40 & 15 & 32.6 & 42 & -22.4 & & 51.9 & 50 & 3.8 & \\
\hline 60 & 3 & 22.8 & 26 & -12.3 & & 33.6 & 35 & -4.0 & \\
\hline 60 & 5 & 28.95 & 34 & -14.9 & & 45.4 & 48 & -5.4 & \\
\hline 60 & 10 & $33.1^{\dagger}$ & 40 & -17.3 & & $56.35^{\dagger}$ & 54 & 4.4 & \\
\hline 60 & 15 & 48.6 & 48 & 1.3 & & 75 & 61 & 23.0 & \\
\hline 80 & 10 & 21.7 & 46 & -52.8 & & 41.2 & 66 & -37.6 & \\
\hline 80 & 15 & 43.5 & 55 & -20.9 & & 68.5 & 73 & -6.2 & \\
\hline \multicolumn{10}{|l|}{ Certus PR } \\
\hline 65 & 5 & 20.05 & 26.4 & -24.1 & $-23.2 \pm 1.2$ & 32.65 & 41.4 & -21.1 & $-17.7 \pm 4.8$ \\
\hline 65 & 10 & 23.75 & 30.6 & -22.4 & & 39.85 & 46.5 & -14.3 & \\
\hline
\end{tabular}

(A)

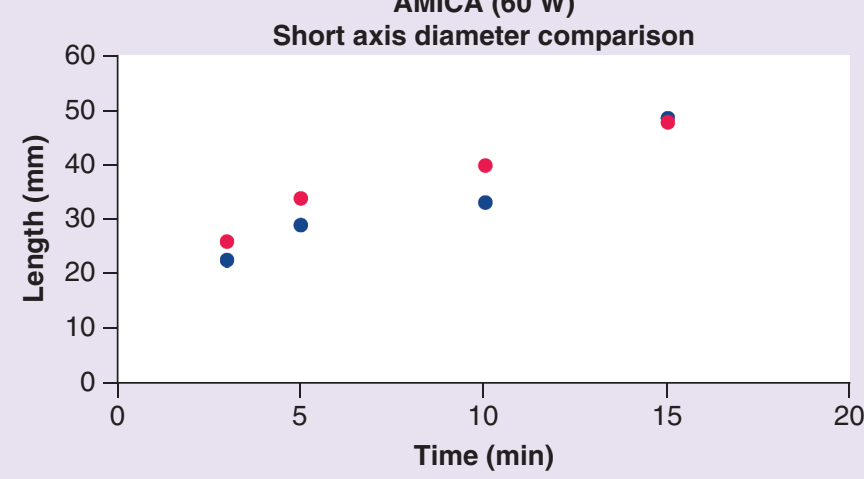

- In vivo human tumor in liver e Ex vivo bovine liver

(C)

Certus PR (65 W)

Short axis diameter comparison

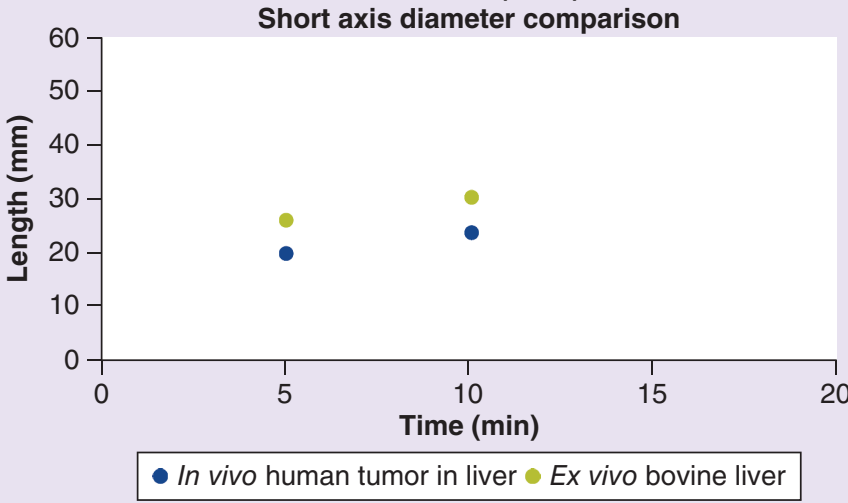

(B)

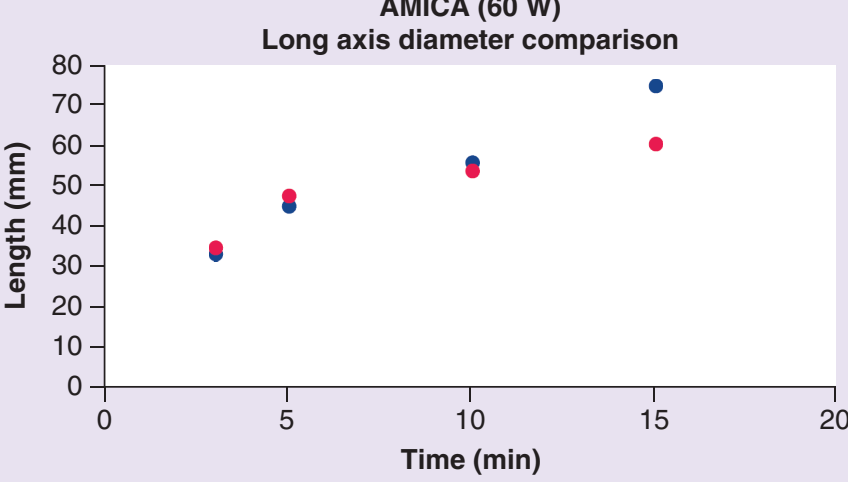

- In vivo human tumor in liver e Ex vivo bovine liver

(D)

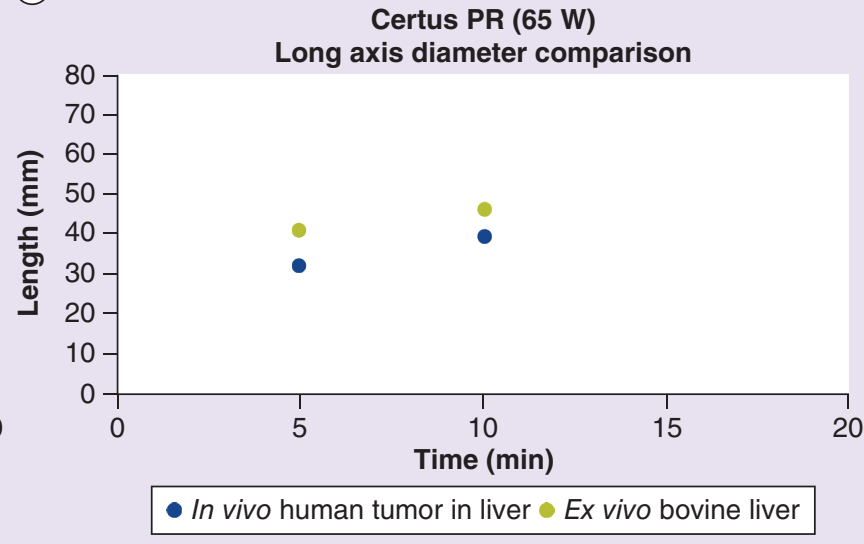

Figure 2. Comparison of in vivo and ex vivo reference ablation diameters for the AMICA and Certus PR applicators at a specified wattage with respect to time. 


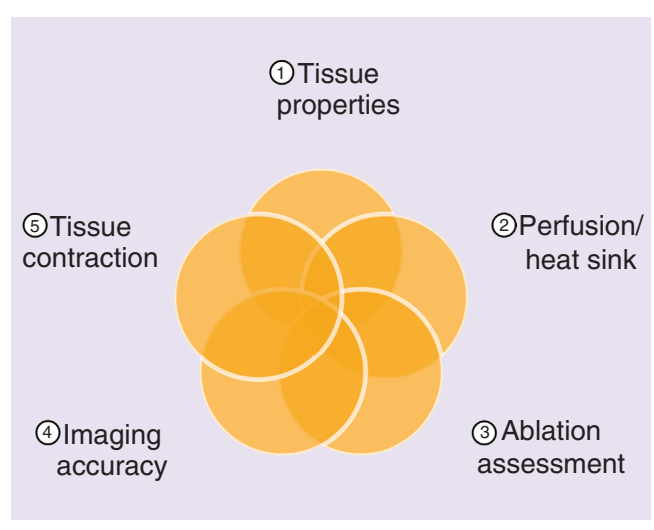

Figure 3. Ex vivo versus in vivo parameters that inherently make accurate comparisons a challenge with the use of MWA technology.

\section{Tissue property impact on MWA volumes}

With multiple parameters to consider in ex vivo versus in vivo studies, as summarized in Figure 3, we must understand the nuances of MWA technology studies to be able to accurately assess the underlying differences and to effectively use this modality clinically. Interestingly but often overlooked, the problem with 'direct comparisons' of ex vivo versus in vivo may be more tissue property related rather than an oversimplified ex vivo versus in vivo difference (e.g., bovine vs porcine vs human, healthy vs diseased tissue, room vs physiological temperature, desiccated vs hydrated tissue, absence or presence of perfusion and central vs peripheral ablations, etc.) $[1,10,28]$.

Ex vivo MWA studies tend to be based on the bovine liver model and in vivo MWA studies tend to be based on the porcine liver model for logistical reasons $[1,27]$. Although the porcine liver model is the gold standard for preclinical studies because it resembles the human liver in size (depending on age/weight), vascularity and metabolic function, the disparate anatomical dimensions of the porcine liver in comparison to the bovine liver (and human liver) may introduce an artificial factor of varying target tissue sizes. Tissue dimensions relative to the expected ablation volume has been shown to alter the size and shape of MWA volumes ex vivo [31]. Thin tissue samples, whose dimensions in the plane perpendicular to the applicator were smaller than the short axis, caused an average increase in ablation lesion length by $7.5 \%$ in comparison to unrestricted samples [31]. Because porcine liver has lobes with a thinner profile than those in human, this study raises concerns about the validity of the in vivo porcine liver model as a gold standard for translational medicine. To corroborate this concern, a study using the AMICA (HS Medical, Rome, Italy) MWA device, showed that ex vivo data on bovine liver were more predictive of the actual clinical performance on centrally located liver malignancies than in vivo data derived from the porcine model [27].

In addition to tissue dimensions affecting MWA volumes, physical features of diseased tissue relative to healthy tissue may contribute to underlying differences in vivo versus ex vivo. For example, malignant tissue ex vivo has been shown to have different electrical tissue properties from normal tissue [10]; this would impact the absorption of microwave energy and heat generation in the varying tissue types. Further, depending on the tumor properties (i.e., hepatocellular carcinoma which tends to occur in cirrhotic livers vs liver metastases), it has been shown that ablation volumes at the same MWA settings were larger in metastases in comparison to hepatocellular carcinoma, most likely attributed to the reduction in water content and subsequent lower absorption of the microwave energy in cirrhotic tissue [27]. How microwave energy is propagated through a tissue will not only be dependent on the specific MWA device but also on the specific tissue that is to be treated.

\section{Perfusion/heat sink effect on MWA volumes}

One of the key differences of ex vivo and in vivo studies for MWA technologies is the absence or presence of vascular perfusion and the heat sink effect. The heat sink effect is a phenomenon that limits the effectiveness of the ablation when the target lesion is in close proximity (within $1 \mathrm{~cm}$ ) to a large blood vessel ( $\geq 3 \mathrm{~mm}$ in diameter); the flowing blood causes a cooling effect, thereby reducing the ablation volume [13]. In vivo MWA volumes are generally smaller than ex vivo MWA volumes and this disparity is mainly attributed to the heat sink effect [1,12]. With the $915 \mathrm{MHz}$ VivaWave MWA System (Vivant Medical, CA, USA) at $45 \mathrm{~W}$ for 5-10 min, MWA has been shown to have less susceptibility to the heat sink effect in an in vivo porcine model compared with previous data reported with RFA $[8,13]$. Given that computational modeling shows that perfusion has greater effects on ablation diameter with respect to antennas operating at $915 \mathrm{MHz}$ versus $2.45 \mathrm{GHz}$, greater effective conductivity and power 
deposition associated with MWA systems operating at $2.45 \mathrm{GHz}$ may overcome the effects of perfusion even more so than previously proposed [1,8]. In agreement with this, with the $2.45 \mathrm{GHz}$ Microsulis MTA System (Microsulis Medical) at higher power settings of 100 and $150 \mathrm{~W}$, in vivo short axis diameters for treatment durations of 8 min or less were unconventionally larger than for ex vivo short axis diameters [1]. MWA technology is generally less susceptible to perfusion and the heat sink effect than RFA, but depending on the power delivery (i.e., operating frequency, MWA applicator design, power and time settings), the severity of the heat sink effect experienced in vivo may contribute to differences in MWA volumes obtained from ex vivo animal studies.

Although there are clinical studies supporting the efficient and safe use of MWA technology in treating liver tumors adjacent to large vessels [32-34], Vogl et al.'s comparison of a $915 \mathrm{MHz}$ (Evident, CO, USA) and a $2.45 \mathrm{GHz}$ (Emprint, Covidien, CO, USA) MWA systems for the treatment of lung metastases, showed that the perivascular location of a tumor was positively associated with local tumor progression [35]. Further, ablations performed with the $915 \mathrm{MHz}$ Evident System led to more local tumor progression than with the use of the $2.45 \mathrm{GHz}$ Emprint System due to the larger ablations and hence sufficiently larger safety margins associated with the high frequency MWA system [35]. Given the many options of commercially available MWA systems, the ability of a specific MWA system to be able to overcome the effects of perfusion will be dependent on the frequency, applicator design and power output that will be unique to each manufacturer.

\section{Imaging challenges in assessing MWA volumes}

While ex vivo ablation volumes are easier to quantitate, ablation assessment is not standardized in freshly ablated in vivo tissue because of the hyperemic zone, unclear margins of cell death and the kinetics of the dynamic ablation zone with time. For instance, some studies include the hyperemic zone which may contain viable cells, and this may make comparing ablation volumes fairly across MWA technologies even more challenging, leading to inconsistencies discerning the differences between ex vivo versus in vivo data [14,36]. In a prospective ablate and resect study, the zone of cell death, which was assessed by triphenyl tetrazolium chloride (TTC) staining, was always greater than the macroscopic gross measurement by approximately $25 \%$ [15]. Of note, the same study that was able to detect larger in vivo ablation volumes compared with ex vivo ablation volumes at high wattages also utilized TTC staining, which may be a more sensitive assay for necrosis than gross margin assessments using calipers [1]. In another study, nicotinamide adenine dinucleotide $(\mathrm{NADH})$ diaphorase staining over hematoxylin and eosin staining provided definitive delineation of the coagulation margins for fresh in vivo ablations [8]. A possible reason for the difficulty to discern the margins in freshly ablated tissue in vivo may be due to the gradual expansion of the ablation zone that has been reported to occur up to 12-h post ablation [16].

Also, the assumption that imaging findings are equivalent to pathological findings must be carefully assessed $[14,17-$ 19]. As noted by Correa-Gallego et al., ultrasound may provide gross estimations of ablation volumes but is unable to predict the degree of cellular injury by significantly underestimating the real ablation volume in the in vivo porcine model [17]. Furthermore, in the human model, the same study showed that ultrasound grossly underestimated the ablation volume compared with delayed computed tomography (CT) scans, emphasizing the limitations of imaging modalities and the temporal contributions of ongoing cellular injury and repair to realized ablation volumes. CT may provide a rough ablation zone estimate but on average, differed by $4 \mathrm{~mm}$ from gross lesion measurement in Awad et al.'s study [19]. Imaging modality and delayed imaging seem to consistently play a critical role in assessing realized ablation volumes because a $24 \mathrm{~h}$ delayed magnetic resonance imaging (MRI) study showed a $24.3 \%$ increase in mean MWA volume in comparison to CT [16-18]; whether this increase was mainly due to the limitation of imaging modality, enlargement of the zone of coagulated necrosis with time and/or rehydration of contracted tissue and to what extent each factor contributes to the overall assessment will have to be further investigated.

\section{Tissue contraction influence on short axis diameter assessment}

Finally, one of the most important parameters that is often overlooked in assessing post-treatment visualized ablation volumes is tissue contraction. Tissue contraction can be caused by dehydration and collapse of tissue architecture during the course of MWA. Because MWA results in very high temperatures of greater than $150^{\circ} \mathrm{C}$, MWA associated tissue contraction in ex vivo bovine liver along the short axis diameter has been shown to be significantly more pronounced than with RFA, 30 and 15\%, respectively, primarily due to tissue dehydration [20]. In addition, the greater degree of contraction experienced with ex vivo bovine lung of $49-55 \%$ along the short axis diameter highlights the importance of taking into consideration the tissue architecture and composition that may be compromised with thermal ablation [20]. Tissue contraction correlated with the degree of local dehydration 
caused by MWA has also been confirmed in ex vivo porcine kidneys to cause $26.1-42.1 \%$ underestimation of the effective coagulation volume depending on deployed energy ( $90 \mathrm{~W}$ at 5, 7.5 and $10 \mathrm{~min}$ ) [21]. These ex vivo findings were more recently confirmed in in vivo human tumors with relative contractions around $9.95 \pm 10.4 \%$ using both the Certus 140 LK15 (2.45 GHz) and Evident (915 MHz) Systems (NeuWave Medical; and Medtronic, MN, USA) [22]. However, to better understand how diseased tissue clinically is affected by applied energies from differing MWA systems, the degree of contraction should have been noted per MWA device especially because of the different operating frequencies. More studies will be needed to carefully elucidate the impact of generator operating frequency and specific microwave applicator design and settings (power and time) on the degree of tissue contraction experienced in clinical applications.

Taking tissue contraction into consideration is very important because microwave tissue ablation is a dynamic process and by not taking this phenomenon into account, the clinician may mistakenly assume that the treated volume was insufficient and risk overcompensating with additional applied MWA energy. This would result in unnecessarily destroying healthy tissue in an otherwise potentially compromised patient. The clinician should be aware of tissue contraction as it relates to the specific MWA device and settings in addition to the treating tissue properties. Qualitative differences in tissue desiccation has been reported in vivo with higher power settings of 100 $\mathrm{W}$ causing more complete desiccation and charring over 50 and $25 \mathrm{~W}$ at the same total applied energy of $30 \mathrm{~kJ}[30]$. Additionally, ablation volumes in cirrhotic livers have been shown to be slightly larger than in noncirrhotic livers, suggesting that fibrosis and reduced water content may render certain tissues less susceptible to tissue contraction [29]. To what degree tissue contraction in combination with distinct tissue properties and perfusion all play a role in determining actual MWA volumes in vivo will be vital in understanding and effectively using this newer and exciting ablation technology.

\section{Conclusion \& future perspective}

In summary, the ability to effectively translate ex vivo based MWA results in vivo clinically with high fidelity has not yet been fully potentiated because every MWA device is unique and clinical results will depend on the specific MWA device being utilized and the underlying tissue characteristics. Ex vivo data provided by manufacturers may serve as gross approximations of ablation volumes in their respective in vivo human organ counterparts, but the interplay of how tissue properties, perfusion and the heat sink effect, ablation volume assessment, imaging accuracy and tissue contraction all contribute to realized ablation volumes clinically is still under early investigation especially at particular wattage and time settings per MWA device. This review highlights parameters that investigators have started to elucidate with the adoption of MWA technology. By being cognizant of these factors, clinicians should be inspired to formulate additional testable hypotheses to accelerate the standardization of MWA technologies in the clinic to ultimately maximize patient outcomes in a more predictive manner.

\section{Acknowledgements}

The author would like to acknowledge S Dietz, Global Director of Oncology/Surgery at AngioDynamics, for identifying the need for a publication to address ex vivo and in vivo differences and for providing helpful feedback. The author would also like to acknowledge DM Liu, Clinical Associate Professor, Faculty of Medicine, at University of British Columbia for reviewing the paper and encouraging submission to Future Oncology for publication.

\section{Financial \& competing interests disclosure}

$\mathrm{C}$ Kim is an employee of AngioDynamics. The author has no other relevant affiliations or financial involvement with any organization or entity with a financial interest in or financial conflict with the subject matter or materials discussed in the manuscript apart from those disclosed.

No writing assistance was utilized in the production of this manuscript.

\section{Open access}

This work is licensed under the Attribution-NonCommercial-NoDerivatives 4.0 Unported License. To view a copy of this license, visit http://creativecommons.org/licenses/by-nc-nd/4.0/ 
Executive summary

Microwave ablation is a different thermal ablation than radiofrequency ablation

- Microwave ablation (MWA) and radiofrequency ablation (RFA) achieve different energy deposition and kinetics; therefore, MWA may need to be assessed differently than previously assumed with RFA technology.

Each MWA system is uniquely different

- Because MWA technologies differ in design and energy deposition, generalizations about MWA technologies must be empirically defined per device and not misleadingly across all similarly classified technologies.

Ex vivo MWA validations versus in vivo MWA results

- Ablation dimensions vary in vivo versus ex vivo and to what extent will depend on the MWA system's specific applicator design, power and time settings, and tissue properties.

Tissue property impact on MWA volumes

- Differences in tissue properties may contribute to differences in realized ablation volumes in clinical practice from manufacturer's reference MWA volumes.

Perfusion/heat sink effect on MWA volumes

- MWA technology is generally less susceptible to the heat sink effect than RFA but the ability to overcome perfusion mediated cooling will be dependent on the MWA system's design and power output.

Imaging challenges in assessing MWA volumes

- Imaging modality and delayed imaging seem to consistently play a critical role in assessing realized ablation volumes.

Tissue contraction influence on short axis diameter assessment

- Tissue contraction can be caused by dehydration and collapse of tissue architecture during the course of MWA and the degree of contraction will be dependent on both the tissue and the MWA device protocol.

Conclusion

- Factors to consider for more accurate MWA assessments are tissue properties, perfusion/heat sink, ablation assessment, imaging accuracy and tissue contraction when assessing post-treatment MWA volumes in comparison to manufacturer's ex vivo ablation volumes to ensure that the anticipated ablation volumes closely approximates the actual treatment volumes for better patient outcomes.

- Ex vivo data may serve as gross approximations of ablation volumes in their respective in vivo human organ counterparts but the interplay of how tissue properties, perfusion/heat sink effect, ablation assessment, imaging accuracy and tissue contraction contribute to realized ablation volumes clinically is still under early investigation especially at particular wattage and time settings per MWA device.

\section{References}

Papers of special note have been highlighted as: $\bullet$ of interest; $\bullet \bullet$ of considerable interest

1. Hines-Peralta AU, Pirani N, Clegg P et al. Microwave ablation: results with a 2.45-GHz applicator in ex vivo bovine and in vivo porcine liver. Radiology 239(1), 94-102 (2006).

-. Compares in vivo and ex vivo microwave ablation (MWA) volume differences based on power and time settings.

2. Simo KA, Tsirline VB, Sindram D et al. Microwave ablation using $915-\mathrm{MHz}$ and $2.45-\mathrm{GHz}$ systems: what are the differences? $H P B$ (Oxford) 15(12), 991-996 (2013).

3. Wright AS, Sampson LA, Warner TF, Mahvi DM, Lee FT Jr. Radiofrequency versus microwave ablation in a hepatic porcine model. Radiology 236(1), 132-139 (2005).

4. Lubner MG, Brace CL, Hinshaw JL, Lee FT Jr. Microwave tumor ablation: mechanism of action, clinical results, and devices. J. Vasc. Interv. Radiol. 21(8 Suppl.), S192-S203 (2010).

- Provides an excellent review on MWA mechanism of action.

5. Hong K, Georgiades C. Radiofrequency ablation: mechanism of action and devices. J. Vasc. Interv. Radiol. 21(8 Suppl.), S179-S186 (2010).

- Provides an excellent review on radiofrequency ablation (RFA) mechanism of action.

6. Schramm W, Yang D, Haemmerich D. Contribution of direct heating, thermal conduction and perfusion during radiofrequency and microwave ablation. Conf. Proc. IEEE Eng. Med. Biol. Soc. 1, 5013-5016 (2006).

7. Ringe KI, Lutat C, Rieder C, Schenk A, Wacker F, Raatschen HJ. Experimental evaluation of the heat sink effect in hepatic microwave ablation. PLoS ONE 10(7), e0134301 (2015).

8. Yu NC, Raman SS, Kim YJ, Lassman C, Chang X, Lu DS. Microwave liver ablation: influence of hepatic vein size on heat-sink effect in a porcine model. J. Vasc. Interv. Radiol. 19(7), 1087-1092 (2008).

-. Shows how hepatic vein size affects MWA in an in vivo porcine liver model. 
9. Brace CL. Radiofrequency and microwave ablation of the liver, lung, kidney, and bone: what are the differences? Curr. Probl. Diagn. Radiol. 38(3), 135-143 (2009).

10. O'rourke AP, Lazebnik M, Bertram JM et al. Dielectric properties of human normal, malignant and cirrhotic liver tissue: in vivo and ex vivo measurements from 0.5 to $20 \mathrm{GHz}$ using a precision open-ended coaxial probe. Phys. Med. Biol. 52(15), 4707-4719 (2007).

11. Curto S, Taj-Eldin M, Fairchild D, Prakash P. Microwave ablation at $915 \mathrm{MHz}$ vs $2.45 \mathrm{GHz}$ : a theoretical and experimental investigation. Med. Phys. 42(11), 6152-6161 (2015).

12. Siriwardana PN, Singh S, Johnston EW et al. Effect of hepatic perfusion on microwave ablation zones in an ex vivo porcine liver model. J. Vasc. Interv. Radiol. 28(5), 732-739 (2016).

13. Goldberg SN, Hahn PF, Tanabe KK et al. Percutaneous radiofrequency tissue ablation: does perfusion-mediated tissue cooling limit coagulation necrosis? J. Vasc. Interv. Radiol. 9(1 Pt 1), 101-111 (1998).

14. Goldberg SN, Charboneau JW, Dodd GD 3rd et al. Image-guided tumor ablation: proposal for standardization of terms and reporting criteria. Radiology 228(2), 335-345 (2003).

15. Ratanaprasatporn L, Charpentier KP, Resnick M, Lu S, Dupuy D. Intra-operative microwave ablation of liver malignancies with tumour permittivity feedback control: a prospective ablate and resect study. HPB (Oxford) 15(12), 997-1001 (2013).

16. Ohno T, Kawano K, Sasaki A, Aramaki M, Yoshida T, Kitano S. Expansion of an ablated site and induction of apoptosis after microwave coagulation therapy in rat liver. J. Hepatobiliary Pancreat. Surg. 8(4), 360-366 (2001).

17. Correa-Gallego C, Karkar AM, Monette S, Ezell PC, Jarnagin WR, Kingham TP. Intraoperative ultrasound and tissue elastography measurements do not predict the size of hepatic microwave ablations. Acad. Radiol. 21(1), 72-78 (2014).

18. Ringe KI, Wacker F, Raatschen HJ. Is there a need for MRI within 24 hours after CT-guided percutaneous thermoablation of the liver? Acta Radiol. 56(1), 10-17 (2015).

19. Awad MM, Devgan L, Kamel IR, Torbensen M, Choti MA. Microwave ablation in a hepatic porcine model: correlation of CT and histopathologic findings. HPB (Oxford) 9(5), 357-362 (2007).

20. Brace CL, Diaz TA, Hinshaw JL, Lee FT Jr. Tissue contraction caused by radiofrequency and microwave ablation: a laboratory study in liver and lung. J. Vasc. Interv. Radiol. 21(8), 1280-1286 (2010).

-• Compares tissue contraction using RFA and MWA in ex vivo liver and lung.

21. Sommer CM, Sommer SA, Mokry T et al. Quantification of tissue shrinkage and dehydration caused by microwave ablation: experimental study in kidneys for the estimation of effective coagulation volume. J. Vasc. Interv. Radiol. 24(8), 1241-1248 (2013).

22. Lee JK, Siripongsakun S, Bahrami S, Raman SS, Sayre J, Lu DS. Microwave ablation of liver tumors: degree of tissue contraction as compared to RF ablation. Abdom. Radiol. (NY) 41(4), 659-666 (2016).

\section{-. Compares tissue contraction using RFA and MWA in liver tumors.}

23. Vogl TJ, Hagar A, Nour-Eldin NA et al. High-frequency versus low-frequency microwave ablation in malignant liver tumours: evaluation of local tumour control and survival. Int. J. Hyperthermia 32(8), 868-875 (2016).

24. Hoffmann R, Rempp H, Erhard L et al. Comparison of four microwave ablation devices: an experimental study in ex vivo bovine liver. Radiology 268(1), 89-97 (2013).

-. Provides an excellent comparison of MWA devices in an ex vivo model.

25. Saccomandi P, Schena E, Massaroni $\mathrm{C}$ et al. Temperature monitoring during microwave ablation in ex vivo porcine livers. Eur. J. Surg. Oncol. 41(12), 1699-1705 (2015).

26. Harari CM, Magagna M, Bedoya $M$ et al. Microwave ablation: comparison of simultaneous and sequential activation of multiple antennas in liver model systems. Radiology 278(1), 95-103 (2016).

27. Amabile C, Ahmed M, Solbiati L et al. Microwave ablation of primary and secondary liver tumours: ex vivo, in vivo, and clinical characterisation. Int. J. Hyperthermia 33(1), 34-42 (2017).

28. Winokur RS, Du JY, Pua BB et al. Characterization of in vivo ablation zones following percutaneous microwave ablation of the liver with two commercially available devices: are manufacturer published reference values useful? J. Vasc. Interv. Radiol. 25(12), 1939e1931-1946e1931 (2014).

29. Shyn PB, Bird JR, Koch RM et al. Hepatic microwave ablation zone size: correlation with total energy, net energy, and manufacturer-provided chart predictions. J. Vasc. Interv. Radiol. 27(9), 1389-1396 (2016).

30. Bedoya M, Del Rio AM, Chiang J, Brace CL. Microwave ablation energy delivery: influence of power pulsing on ablation results in an $e x$ vivo and in vivo liver model. Med. Phys. 41(12), 123301 (2014).

31. Cavagnaro M, Amabile C, Cassarino S, Tosoratti N, Pinto R, Lopresto V. Influence of the target tissue size on the shape of ex vivo microwave ablation zones. Int. J. Hyperthermia 31(1), 48-57 (2015).

32. Yu J, Liang P, Yu XL et al. Local tumour progression after ultrasound-guided microwave ablation of liver malignancies: risk factors analysis of 2529 tumours. Eur. Radiol. 25(4), 1119-1126 (2015).

33. Dou JP, Yu J, Yang XH et al. Outcomes of microwave ablation for hepatocellular carcinoma adjacent to large vessels: a propensity score analysis. Oncotarget 8(17), 28758-28768 (2017). 
34. Huang S, Yu J, Liang P et al. Percutaneous microwave ablation for hepatocellular carcinoma adjacent to large vessels: a long-term follow-up. Eur. J. Radiol. 83(3), 552-558 (2014).

35. Vogl TJ, Roman A, Nour-Eldin NA, Hohenforst-Schmidt W, Bednarova I, Kaltenbach B. A comparison between $915 \mathrm{MHz}$ and 2450 $\mathrm{MHz}$ microwave ablation systems for the treatment of small diameter lung metastases. Diagn. Interv. Radiol. 24(1), 31-37 (2018).

36. Howk K, Ladtkow C, Peterson D, Cafaro A. Consistent and predictable spherical ablation shape in both liver and lung: performance of the emprint ablation system with thermosphere technology in an in vivo porcine model. [Poster 129]. J. Vasc. Interv. Radiol. 26(5), e89-e90 (2015). 